\title{
Spectroscopic Signatures of AA' and AB Stacking of Chemical Vapor Deposited Bilayer $\mathrm{MoS}_{2}$
}

\section{(Supporting Information)}

Ming Xia, ${ }^{1}$ Bo Li, ${ }^{2}$ Kuibo Yin, ${ }^{3,4}$ Giovanni Capellini, ${ }^{5}$ Gang Niu,,${ }^{5}$ Yongji Gong, ${ }^{2}$ Wu

Zhou, ${ }^{4}$ Pulickel M.Ajayan, ${ }^{2}$ Ya-Hong Xie $e^{1 *}$

1. Department of Materials Science and Engineering, University of California, Los Angeles, Los Angeles, California 90095, United States

2. Department of Materials Science and NanoEngineering, Rice University, Houston, Texas 77005, USA

3. SEU-FEI Nano-Pico Center, Key Laboratory of MEMS of Ministry of Education, Southeast University, Nanjing 210096, China

4. Materials Science and Technology Division, Oak Ridge National Laboratory, Oak Ridge, Tennessee 37831, USA

5. Innovations for High Performance (IHP), ImTechnologiepark 25, Frankfurt (Oder) D-15236, Germany

1. Au nano-pyramid fabrication 
Au nano-pyramids were fabricated using sphere lithography method. ${ }^{1}$ Figure S1 shows the fabrication process. Because Si surface was not fully covered by polystyrene spheres during spin coating at the beginning, part of the Au substrate did not contain Au pyramids, i.e. being flat as shown in Figure S2.

(1) Spin coating polystyrene(PS) spheres (500 $\mathrm{nm}$ diameter) on Si substrate

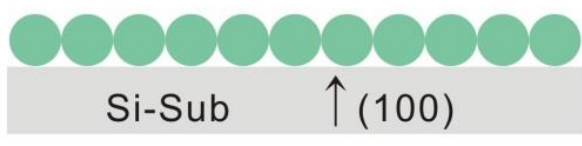

(2) O2 plasma etch PS spheres

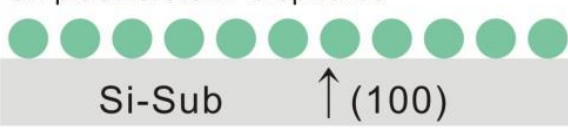

(3) Deposit SiO2 film to form hard mask

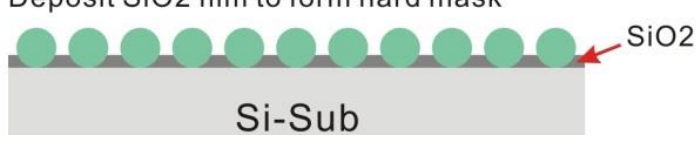

(4) $60 \mathrm{wt} \% \mathrm{KOH}$ wet etching at $60 \mathrm{C}$

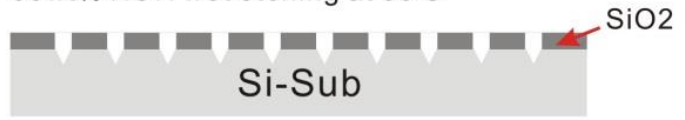

(5) HF dipping to remove $\mathrm{SiO} 2$ and deposit $\mathrm{Au}$ on bare pitted Si substrate

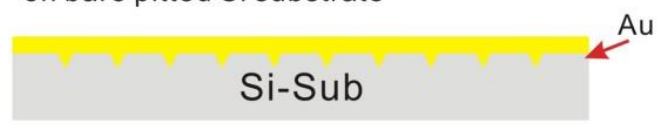

(6) Peel off the Au film using epoxy

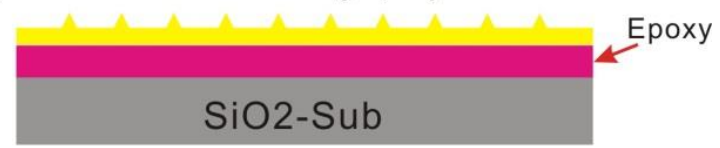

Figure S1. Fabrication process of Au nano-pyramid substrates.

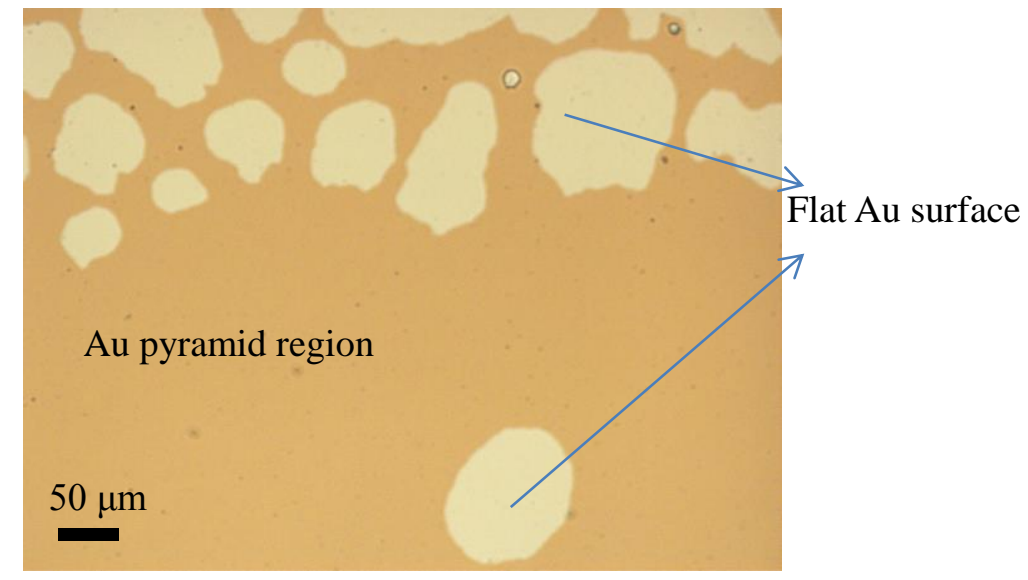

Figure S2. Optical image of Au tipped substrates.

2. Raman data analysis

Raman data was analyzed using Renishaw WiRE 3.2 software. The background of the Raman spectra was removed using polynomial fitting algorithm. Figure S3 (a) shows the Raman spectral background of bare Au pyramids. Figure S3 (b) and (c) show the original Raman spectrum ( $\mathrm{AA}^{\prime}$ stacked bilayer $\mathrm{MoS}_{2}$ ) and the one after background removal, respectively. Peak selection and fitting were conducted using the "Peak pick" and "Curve fit" functions in 
WiRE 3.2 software.
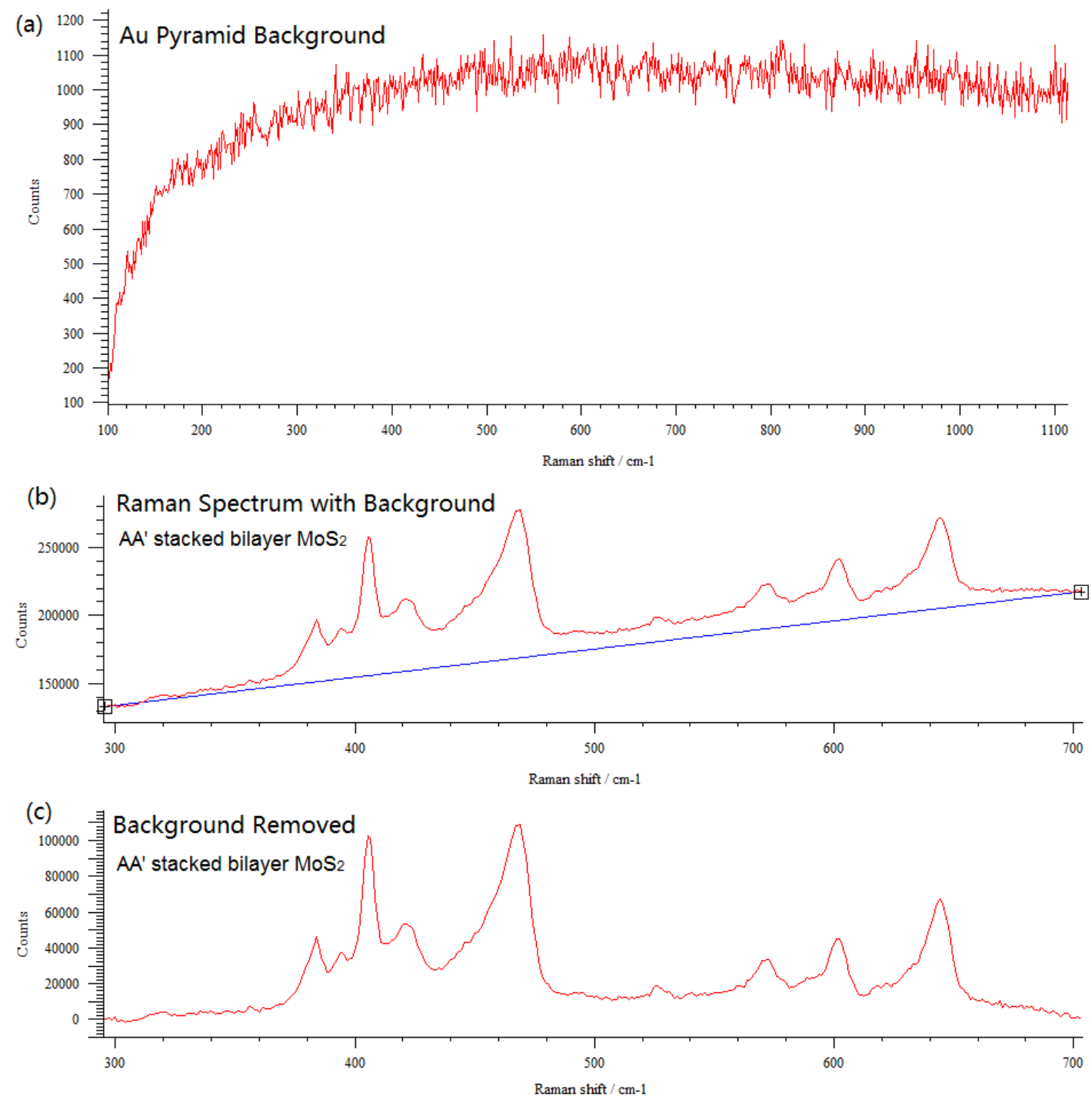

Figure S3 (a) The Raman spectrum of bare Au tipped substrates. (b) The original Raman spectrum of AA' stacked bilayer $\mathrm{MoS}_{2}$ on Au tips. (c) The Raman spectrum in (b) after background removal. 

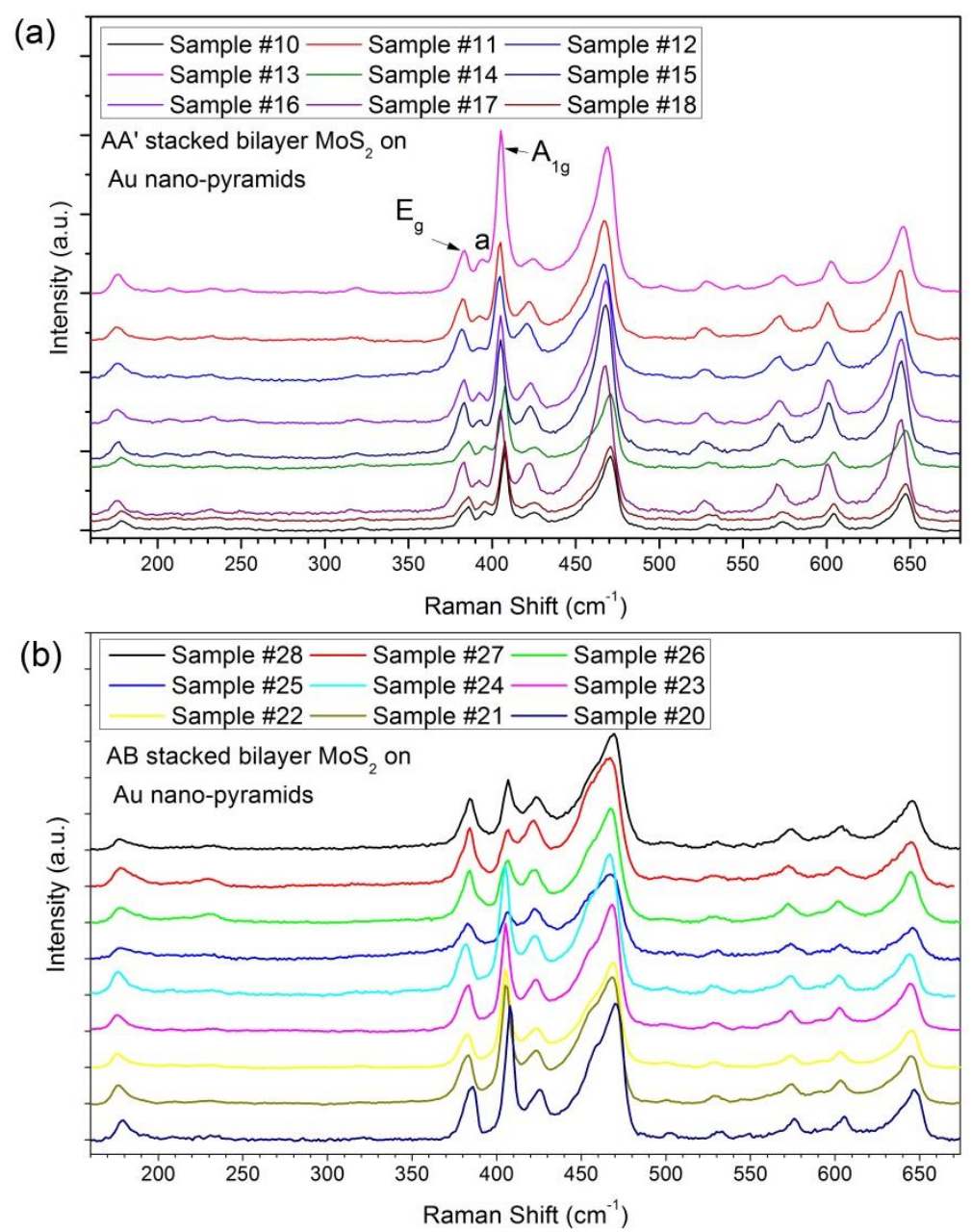

Figure S4. (a) Resonance Raman spectra of AA' stacked bilayer $\mathrm{MoS}_{2}$ measured from 9 samples. (b) Resonance Raman spectra of $\mathrm{AB}$ stacked bilayer $\mathrm{MoS}_{2}$ measured from 9 samples. The " $\mathrm{a}$ " peak is present in all of the AA' stacked bilayer samples but in none of the AB stacked bilayer samples. No exception was found for all the bilayer samples we measured.

\section{SERS enhancement factor for bilayer $\mathrm{MoS}_{2}$}

While the signal of conventional Raman spectra being derived from an area comparable to the excitation wavelength, Raman signals from plasmonic enhancement comes predominantly from electromagnetic field concentration that is extremely localized to typically nanometer region (hotspot). The following are the definition of SERS EF we proposed:

$$
\mathrm{EF}=\frac{I(\text { pyramid }) / A(\text { hotspot })}{I(\text { flat }) / A(\text { excitation } \text { spot })}
$$

Where I(pyramid) is the $\mathrm{MoS}_{2}$ Raman peak intensity measured at hotspot on the $\mathrm{Au}$ 
nano-pyramid region. A (hotspot) is the area of a typical hotspot on Au nano-pyramid structure. Our previous study ${ }^{1}$ indicates that the typical size of hotspot of our $\mathrm{Au}$ nano-pyarmid array is about10 nanometer scale. We take A (hotspot) as a 10×10 nm area. I(flat) is the $\mathrm{MoS}_{2}$ Raman peak intensity measured at flat $\mathrm{Au}$ film or flat $\mathrm{SiO}_{2} / \mathrm{Si}$ substrate. The A(excitation spot) is the area of the laser excitation spot on flat region. We used $100 \times$ lens with NA 0.9 for $633 \mathrm{~nm}$ laser, and the calculated laser spot size is $\sim 860 \mathrm{~nm}$. We take $\mathrm{A}($ excitation spot) as a $860 \times 860 \mathrm{~nm}$ area.

We choose Eg peak ( $385 \mathrm{~cm}-1)$ intensity as $\mathrm{MoS}_{2}$ Raman peak intensity in the equation (1) to calculate SERS EF because this peak can be clearly identified in Au nano-pyramid, flat $\mathrm{Au}$ and $\mathrm{SiO}_{2} / \mathrm{Si}$ substrates. Table $\mathrm{S} 1$ shows the SERS EF of bilayer $\mathrm{MoS}_{2}$ on Au nano-pyramid relative to flat $\mathrm{Au}$ surface and $\mathrm{SiO}_{2} / \mathrm{Si}$ substrate. The SERS EF of bilayer $\mathrm{MoS}_{2}$ is in the order of 105. The SERS EFs for AA' $(2 \mathrm{H})$ and $\mathrm{AB}(3 \mathrm{R})$ stacked bilayer $\mathrm{MoS}_{2}$ are a little different but in the same order of magnitude.

Table S1. SERS EF of bilayer $\mathrm{MoS}_{2}$ on Au nano-pyramid

\begin{tabular}{l|l|l}
\hline & SERS EF relative to flat $\mathrm{Au}$ & SERS EF relative to $\mathrm{SiO}_{2} / \mathrm{Si}$ \\
\hline $\begin{array}{l}\text { AA' stacked }\left(2 \mathrm{H} \text { or } 60^{\circ}\right. \\
\text { stacking) bilayer } \mathrm{MoS}_{2}\end{array}$ & $3.86 \times 10^{5} \pm 1.91 \times 10^{4}$ & $2.11 \times 10^{5} \pm 1.04 \times 10^{4}$ \\
\hline $\begin{array}{l}\mathrm{AB} \text { stacked (3R or 0 } \\
\text { stacking) bilayer } \mathrm{MoS}_{2}\end{array}$ & $3.66 \times 10^{5} \pm 2.22 \times 10^{4}$ & $1.15 \times 10^{5} \pm 0.7 \times 10^{4}$ \\
\hline
\end{tabular}

4. FDTD simulation of Electromagnetic (EM) field distribution around individual $\mathrm{Au}$ pyramids

FDTD simulation was conducted using Lumerical software (FDTD Solutions 8.11). ${ }^{2}$ Figure S5 shows the 3D model used. Au pyramid has a bottom length of $\sim 250 \mathrm{~nm}$ with $\sim 500 \mathrm{~nm}$ pitch. The continuous Au flat film (on the bottom of Au pyramids) thickness is $\sim 20 \mathrm{~nm}$. Figure S6 shows the 3D distribution of the electrical component of the EM-field around individual Au tips. The simulation space is indicated by the yellow cubic shown in Figure S5. Figure S6 (a) shows the 3D distribution of the electrical component of the EM-field symbolized with blue arrows with the cross-sectional views along three major symmetry 
planes being presented in Figure S6 (b-d). Figure S6 (b-d) shows the projections of the electrical component on the Y-Z, X-Z, and X-Y planes, respectively. Red dashed line shows the ridges of the Au pyramid. E-field is not parallel to X-direction near Au pyramid surface. The fact that the electrical component of the EM field possessing significant out-of-plane components can be clearly seen in Figure S6 (b, c).

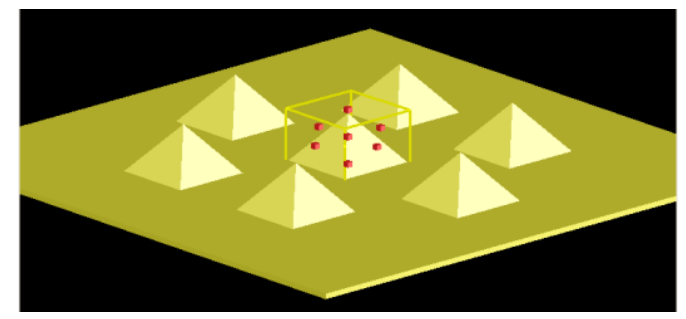

Figure S5.Au pyramid model used for FDTD simulation

(a)

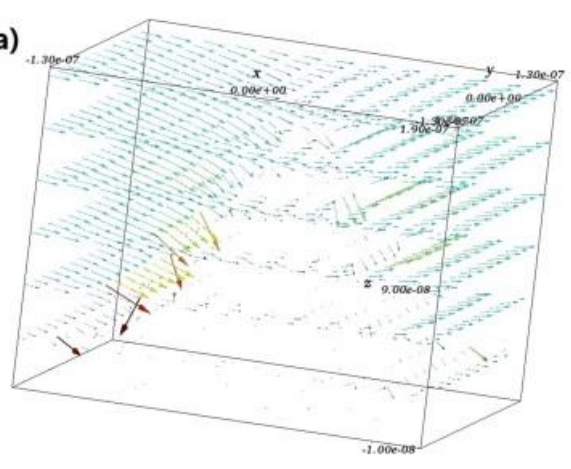

(c)

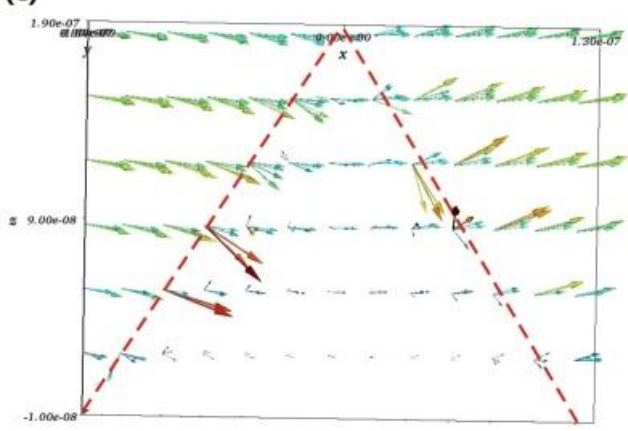

(b)

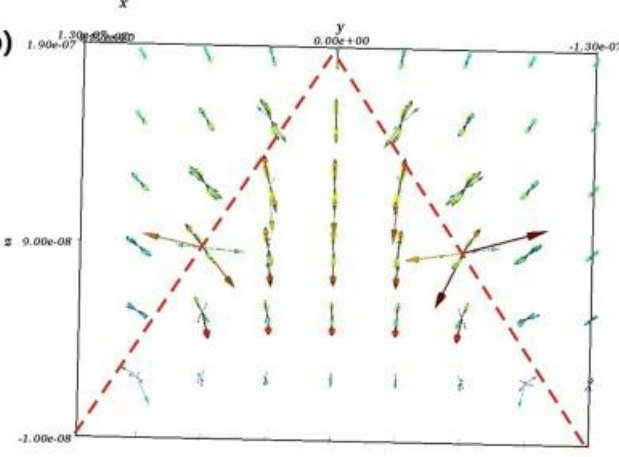

(d)

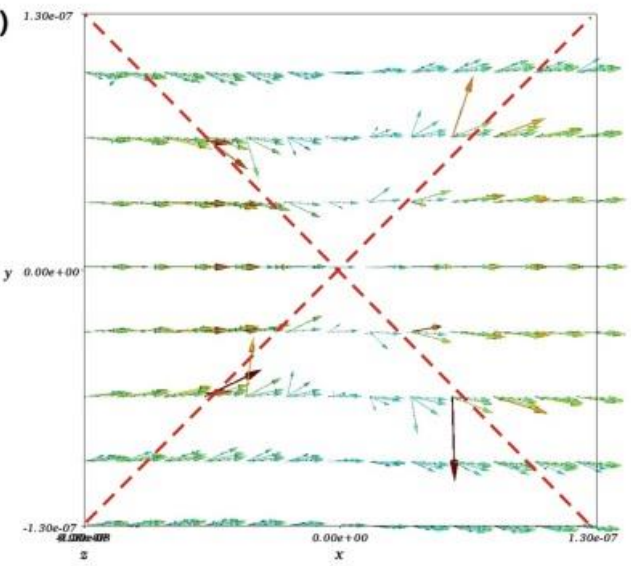

2.3

1.7

1.2

0.58

0.0053

$|E| /\left|E_{0}\right|$

Figure S6. (a) 3D E-field distribution around an Au pyramid. E-field projection on Y-Z (b), X-Z (c), X-Y (d) plane. Red dashed line shows the ridges of Au pyramid. 

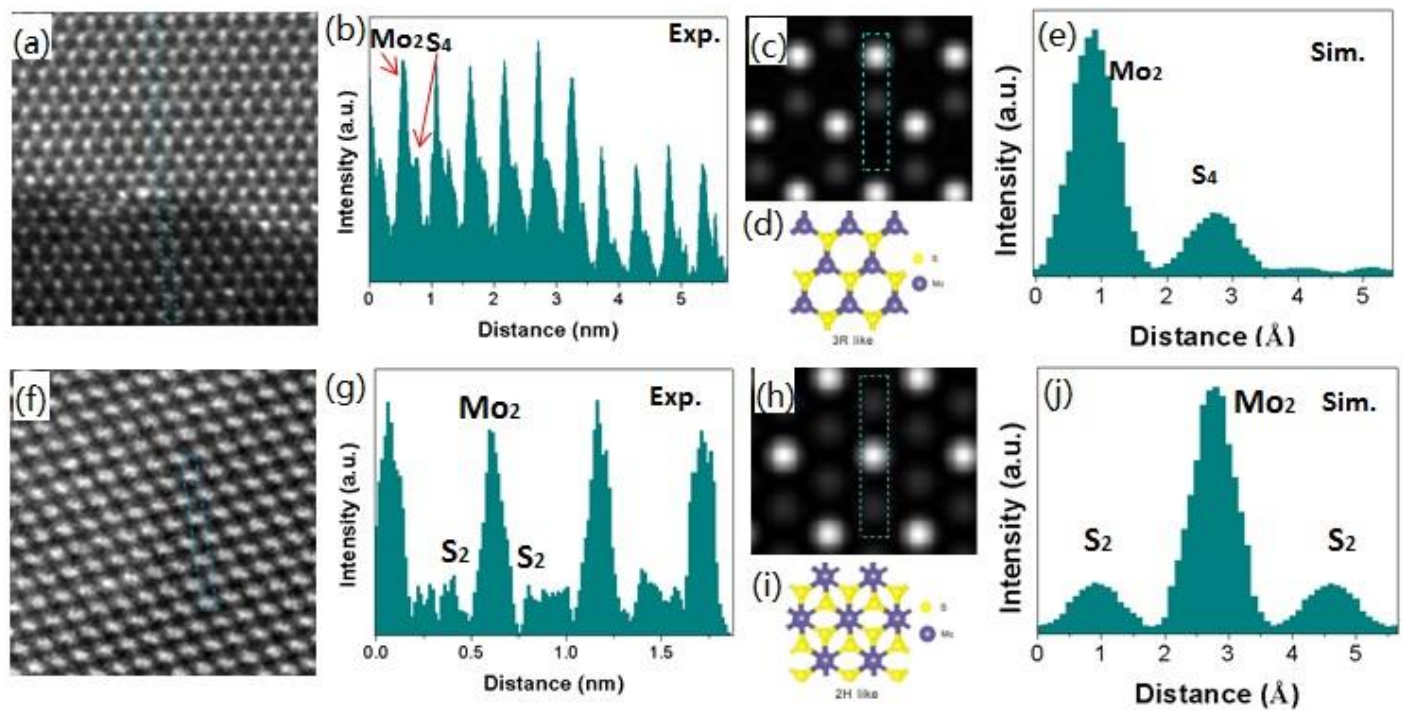

Figure S7 (a) STEM-HAADF image of the AB stacked bilayer $\mathrm{MoS}_{2}$. (b) Intensity profile along the selected line (cyan) in (a). Simulated STEM-HAADF image (c) and the corresponding atomic configuration (d) of bilayer $\mathrm{MoS}_{2}$ of $\mathrm{AB}$ stacking order. (e) Intensity profile along the selected line (bright cyan) in (c). (f) STEM-HAADF image of the AA' stacked bilayer $\mathrm{MoS}_{2}$. (g) Intensity profile along the selected line (cyan) in (f). Simulated STEM-HAADF image (h) and the corresponding atomic configuration (i) of bilayer $\mathrm{MoS}_{2}$ with AA' stacking order. (j) Intensity profile along the selected line (bright cyan) in (h).

(a)

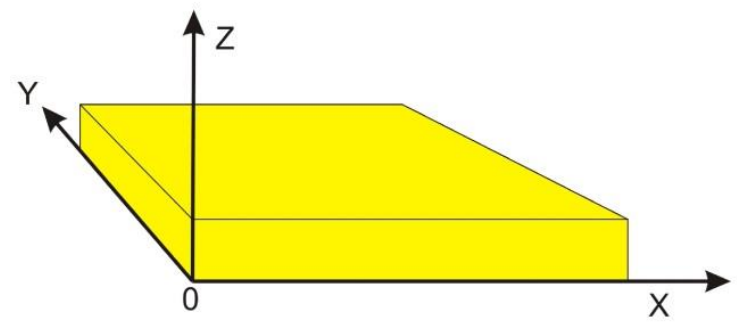

(b)

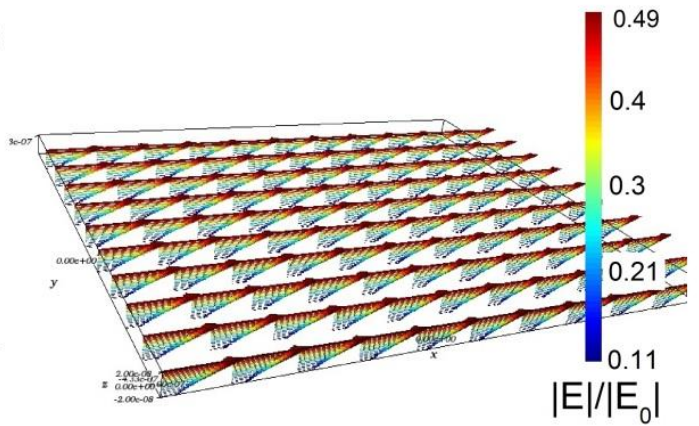

Figure S8. (a) Au flat film model used for FDTD simulation (laser polarization direction is along x axis); (b) 3D E-field distribution on flat Au surface. 


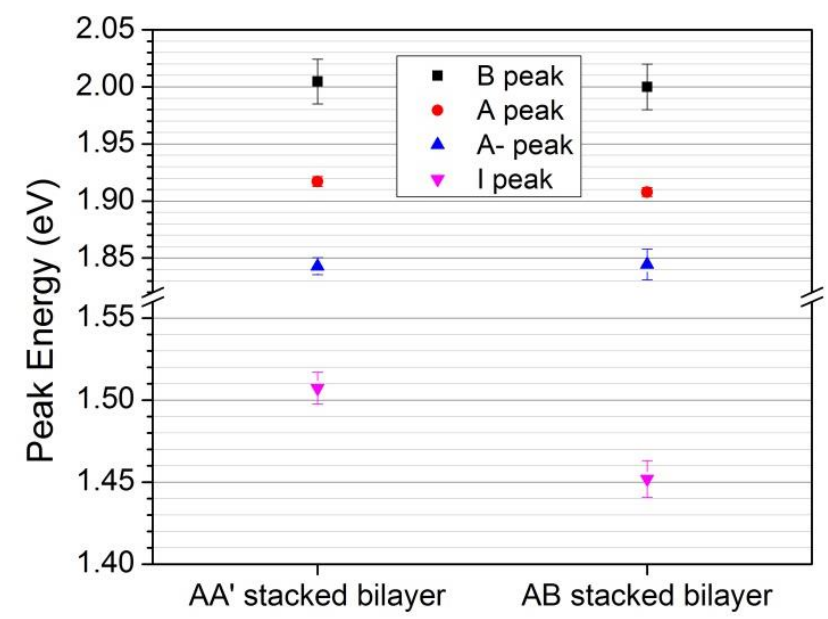

Figure S9 Photoluminescence peak energy of AA' and AB stacked bilayer $\mathrm{MoS}_{2}$ measured from multiple samples on Au nano-pyramids. A noticeable blue shift of the I-peak of AA' stacked bilayer by $0.056 \pm 0.017 \mathrm{eV}$ over that of the $\mathrm{AB}$ stacked bilayer was observed. $\mathrm{A}$ discernable blue shift of the A-peak of AA' stacked bilayer by $0.009 \pm 0.001 \mathrm{eV}$ over that of the AB stacked bilayer was also observed.

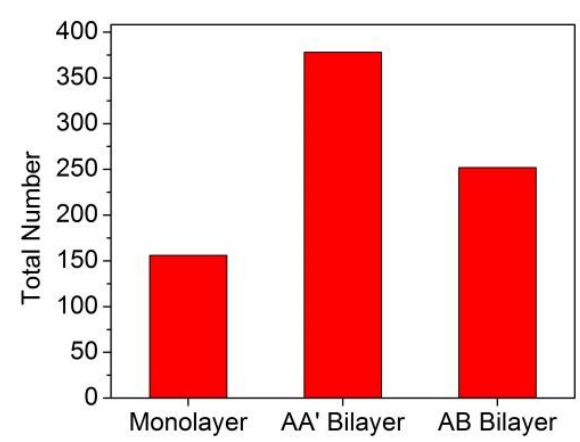

Figure S10. Histogram of CVD grown monolayer, AA' and AB stacked bilayer $\mathrm{MoS}_{2}$.

References:

1. Wang, P.; Zhang, W.; Liang, O.; Pantoja, M.; Katzer, J.; Schroeder, T.; Xie, Y.-H. ACS nano 2012, 6, (7), 6244-6249.

2. https://www.lumerical.com/tcad-products/fdtd/ 